\title{
Clinical shorts
}

Changes in family history over time: Asking about family history can help identify those at increased risk of cancer, but how often should this information be updated? The authors suggest obtaining a comprehensive family history in early adulthood for cancer screening and updating it at least every 5 to 10 years to identify changes in risk. In this cohort study, family histories of breast and colon cancer became increasingly relevant in early adulthood and changed significantly between the ages of 30 and 50 years. Family histories from individuals enrolled in the Cancer Genetics Network, an American registry of those with a personal or family history of cancer, were examined retrospectively from birth until enrolment in the registry, and prospectively from enrolment to last follow-up. Retrospective cancer-specific analyses included between 1817 and 9861 participants, depending on the cancer type (breast, colon or prostate), whereas prospective analyses included between 163 and 1533 participants. Median follow-up was eight years. According to American Cancer Society guidelines, there was a $5 \%$ chance that a person's screening recommendation for colorectal cancer would change between the ages of 30 and 50 years, whereas $4 \%$ of women would become candidates for magnetic resonance imaging screening for breast cancer. See JAMA 2011;306:172-8.

Oxytocin infusion during elective cesarean section: The overall occurrence of major obstetric hemorrhage is not affected by adding an oxytocin infusion to oxytocin bolus after cesarean section, but the need for additional uterotonic agents is reduced. This is the conclusion of a randomized double-blind trial involving 2069 healthy women booked for elective cesarean section at term with a singleton pregnancy. Participants were randomly assigned to receive an oxytocin or normal saline infusion (placebo) after delivery in addition to an oxytocin bolus. There was no difference in the occurrence of major obstetric bleeding of more than $1000 \mathrm{~mL}$ between the treatment and placebo groups (adjusted odds ratio [OR] $0.98,95 \%$ confidence interval $[\mathrm{CI}]$ 0.771.25). The need for additional agents to prevent uterine atony was less in the treatment group (12.2\% v. $18.4 \%$, OR 0.61, 95\% CI 0.48-0.78). Adverse effects, such as nausea, vomiting and hypotension, were similar in both groups. See $B M J$ 2011;343:d4661.

Sertraline or mirtazapine for depression in dementia? A parallel-group double-blind randomized trial recently showed that neither sertraline nor mirtazapine is more effective than placebo for treating depression in patients with dementia. In this study, 326 people with Alzheimer disease and coexisting depression were recruited from outpatient geriatric psychiatry services to receive sertraline, mirtazapine or placebo along with standard care (supportive and problem-solving interventions) for 39 weeks. Severity of depression decreased in all groups compared with baseline at 13 and 39 weeks. However, decreases in depression scores at 13 weeks did not differ between those receiving placebo and those receiving sertraline or mirtazapine. At 39 weeks, there were still no differences between the groups. Adverse events were more common in the sertraline $(43 \%, p=0.010)$ and mirtazapine group $(41 \%, p=0.031)$ than in the placebo group (26\%). There was a large number of withdrawals from all groups. The authors suggest that the decreases in severity of depression may have been related to the standard care provided to the participants in the study, which included a broad range of supportive and problem-solving interventions. See Lancet 2011;378:403-11.

When should parenteral nutrition be started in critically ill adults? Late initiation of parenteral nutrition ( 8 days or more) after admission to an intensive care unit (ICU) may be associated with faster recovery and fewer complications, com- pared with initiation within 48 hours after admission. In this randomized trial in seven ICUs, 4640 patients who were nutritionally at risk were randomized to either early or late initiation of parenteral nutrition aiming to replace $100 \%$ of estimated energy needs; all received an insulin protocol to prevent hyperglycemia. The median length of stay in the ICU for patients in the late initiation group was four days, which was $6 \%$ better than those who had early initiation (hazard ratio 1.06, 95\% confidence interval 1.00-1.13), although this difference was only just statistically significant. Fewer infections and shorter duration of mechanical ventilation and renal replacement therapy in the ICU were also seen in the late initiation group; however, functional status at time of discharge and rates of death in the ICU, in the hospital and at 90 days were similar between the two groups. On the basis of this large trial, early parenteral feeding aimed at replacing complete energy requirements should not be routinely recommended in most critically ill patients. See $N$ Engl J Med 2011;365:506-17.

Are calorie counts on menus accurate? Overall, the stated energy contents of food in sit-down and fast-food restaurants are accurate, but there is substantial inaccuracy for some foods. In this American study, energy counts for 269 food items from 42 restaurants in three states were measured and compared with stated counts on menus. One in five items contained at least $100 \mathrm{kcal}$ per portion more than stated. Particularly in sit-down restaurants, foods with a lower stated energy content systematically contained more energy than stated. One side dish in the study contained $1000 \mathrm{kcal}$ per portion more than the stated energy amount of $450 \mathrm{kcal}$ per portion. See JAMA 2011; 306:287-93.

\section{Diane Kelsall MD MEd \\ Deputy Editor, Clinical Practice CMAJ}

CMAJ 2011. DOI:10.1503/cmaj.111328 